\title{
Indications and timing for tracheostomy in patients with SARS CoV2-related
}

\author{
Emanuele Ferri ${ }^{1}$ (i) . Francesca Boscolo Nata ${ }^{1} \cdot$ Barbara Pedruzzi $^{1}$. Giovanni Campolieti ${ }^{1}$. \\ Francesco Scotto di Clemente ${ }^{1} \cdot$ Fabio Baratto $^{2} \cdot$ Giovanni Cristalli $^{1}$
}

Received: 4 May 2020 / Accepted: 18 May 2020 / Published online: 26 May 2020

(c) Springer-Verlag GmbH Germany, part of Springer Nature 2020

\begin{abstract}
Background The indications and timing for tracheostomy in patients with SARS CoV2-related are controversial.

Purpose In a recent issue published in the European Archives of Otorhinolaryngology, Mattioli et al. published a short communication about tracheostomy timing in patients with COVID-19 (Coronavirus Disease 2019); they reported that the tracheostomy could allow early Intensive Care Units discharge and, in the context of prolonged Invasive Mechanical Ventilation, should be suggested within 7 and 14 days to avoid potential tracheal damages. In this Letter to the Editor we would like to present our experience with tracheostomy in a Hub Covid Hospital.

Methods 8 patients underwent open tracheostomy in case of intubation prolonged over 14 days, bronchopulmonary overlap infections, and patients undergoing weaning. They were followed up and the number and timing of death were recorded.

Results Two patients died after tracheostomy; the median time between tracheostomy and death was 3 days. A negative prognostic trend was observed for a shorter duration of intubation.

Conclusion In our experience, tracheostomy does not seem to influence the clinical course and prognosis of the disease, in the face of possible risks of contagion for healthcare workers. The indication for tracheostomy in COVID-19 patients should be carefully evaluated and reserved for selected patients. Although it is not possible to define an optimal timing, it is our opinion that tracheostomy in a stable or clinically improved COVID-19 patient should not be proposed before the 20th day after orotracheal intubation.
\end{abstract}

Keywords Tracheostomy $\cdot$ SARS CoV2 $\cdot$ COVID-19 $\cdot$ Indications $\cdot$ Timing

The indications and the timing for tracheostomy in patients with SARS CoV2-related are a matter of debate. In the previous Literature, there are several recommendations and Guidelines on "how" to carry out a tracheostomy in COVID-19 patients but, conversely, the indications appear unclear and the timing is variable, essentially referring to

This comment refers to the article available online at https://doi. org/10.1007/s00405-020-05982-0.

Emanuele Ferri

emanuele.ferri@aulss6.veneto.it

1 Otorhinolaryngology Unit, Ospedali Riuniti Padova Sud "Madre Teresa Di Calcutta" Hub Covid Hospital Monselice (Padova)-ULSS 6 Euganea, Padua, Italy

2 Anaesthesia and Intensive Care Unit, Ospedali Riuniti Padova Sud "Madre Teresa Di Calcutta" Hub Covid Hospital Monselice (Padova)-ULSS 6 Euganea, Padua, Italy the experience gained during the previous SARS CoV1 epidemic of 2003 [1].

In a recent issue of European Archives of Otorhinolaryngology, Mattioli et al. published a short communication dealing with the timing of tracheostomy in COVID-19 patients. They report that the tracheostomy reduces the Intensive Care Units (ICUs) stay and, in the context of prolonged invasive mechanical ventilation (IMV), should be suggested within 7 and 14 days to avoid potential tracheal damages [2].

Traditionally, tracheostomy is the commonest surgical procedure in ICUs and is performed to ease weaning from ventilator support, to facilitate airway and pulmonary toilet, to improve patient comfort and daily living activity, to decrease laryngeal injury from endotracheal intubation, and to prevent long-term complications, including tracheal stenosis.

Recently, Shiba et al. reported that, in consideration to the rapid evolution of the disease in critically ill patients, 
tracheostomy does not provide any benefit on the outcome: therefore authors did not believe that prolonged intubation should be considered an indication. Moreover, they reported that current intensive care protocols have a low incidence of tracheal stenosis following a prolonged intubation: this value is not substantially higher than the approximate $2-3 \%$ airway stenosis associated with tracheostomy. Finally, a proven mortality benefit of tracheostomy in severely ill patients in the ICU has not been demonstrated [3].

According to Hiramatsu et al. the optimal timing of tracheotomy in critically ill patients remains controversial. The timing from tracheal intubation to the tracheostomy ranged from 14 to 25 days in previous reports of the SARS pandemic. Considering the unstable general condition in coronavirus patients, early tracheostomy (within 10 days) should be avoided [4].

David et al. underlined that survival in COVID-19 patients requiring IMV is extremely poor $(<20 \%)$ : this argues against early tracheostomy. However, when tracheostomy is indicated authors suggest to delay it from 14 to 21 days post-intubation to allow for sufficient decline in viral load [5].

In the ICU of our Hub Covid Hospital, 8 patients underwent open tracheostomy. Tracheostomy was performed according to the following indications: intubation prolonged over 14 days, bronchopulmonary overlap infections, and patients undergoing weaning. Two patients died after tracheostomy; the median time between tracheostomy and death was 3 days. A negative prognostic trend was observed for a shorter duration of intubation.

In our experience the mortality rate among ICU COVID19 patients undergoing tracheostomy was $25 \%$, a result in line with the previously reported overall mortality rate in ICU COVID-19 patients (26\%). According to our data, we could infer that tracheostomy did not impact on the natural history of these patients.

Another interesting observation is the trend toward significant association between the duration of intubation and overall survival; specifically, in our cohort an intubation shorter than 20 days determined an increased risk of death. This result could support the choice to postpone open tracheostomy in COVID-19 patients, as previously suggested by AAO-HNS for SARS CoV1 patients.

Although it is not possible to define an optimal timing, it is our opinion that tracheostomy in a stable or clinically improved COVID-19 patient should not be proposed before the $20^{\text {th }}$ day after orotracheal intubation.

Funding None.

\section{Compliance with ethical standards}

Conflict of interest The authors declare that they have not conflict of interest.

\section{Reference}

1. Wei WI, Tuen HH, Ng RW, Lam LK (2003) Safe tracheostomy for patients with severe acute respiratory syndrome. Laryngoscope 113:1777-1779

2. Mattioli F, Fermi M, Ghirelli M, Molteni G, Sgarbi N, Bertellini E, Girardis M, Presutti L, Marudi A (2020) Tracheostomy in the COVID-19 pandemic. Eur Arch Otorhinolaryngol. https://doi. org/10.1007/s00405-020-05982-0

3. Shiba T, Ghazizadeh S, Chhetri D, St John M, Long J (2020) Tracheostomy Considerations during the COVID-19 Pandemic. OTO Open. https://doi.org/10.1177/2473974X20922528

4. Hiramatsu M, Nishio N, Ozaki M, Shindo Y, Suzuki K, Yamamoto T, Fujimoto Y, Sone M (2020) Anesthetic and surgical management of tracheostomy in a patient with COVID-19. Auris Nasus Larynx. https://doi.org/10.1016/j.anl.2020.04.002

5. David AP, Russell MD, El-Sayed IH, Russell MS (2020) Tracheostomy guidelines developed at a large academic medical center during the COVID-19 pandemic. Head Neck. https://doi. org/10.1002/hed.26191

Publisher's Note Springer Nature remains neutral with regard to jurisdictional claims in published maps and institutional affiliations. 